\title{
Prognosticators of intravoxel incoherent motion (IVIM) MRI for adverse maternal and neonatal clinical outcomes in patients with placenta accreta spectrum disorders
}

\author{
Tao Lu ${ }^{1}$, Bin Song ${ }^{2}$, Hong Pu ${ }^{1}$, Kui-De Li ${ }^{1}$, Meng-Wei Huang ${ }^{3}$, Jie Mei $^{3}$, Shao-Yu Wang ${ }^{4}$ \\ ${ }^{1}$ Department of Radiology, Sichuan Academy of Medical Sciences \& Sichuan Provincial People's Hospital, Chengdu 610072, China; ${ }^{2}$ Department of \\ Radiology, West China Hospital, Sichuan University, Chengdu 610037, China; ${ }^{3}$ Department of Obstetrics, Sichuan Academy of Medical Sciences \& \\ Sichuan Provincial Peopleal Sciences sChengdu 610072, China; ${ }^{4}$ MR Scientific Marketing Specialist, Siemens Healthineer, Shanghai 201318, China \\ Contributions: (I) Conception and design: T Lu, B Song; (II) Administrative support: B Song, H Pu, SY Wang; (III) Provision of study materials \\ or patients: MW Huang, J Mei; (IV) Collection and assembly of data: T Lu, KD Li; (V) Data analysis and interpretation: T Lu, SY Wang; (VI) \\ Manuscript writing: All authors; (VII) Final approval of manuscript: All authors. \\ Correspondence to: Bin Song. Department of Radiology, West China Hospital, Sichuan University, No. 37, Guoxuexiang, Chengdu 610037, China. \\ Email: songb_radiology@163.com; Hong Pu. Department of Radiology, Sichuan Academy of Medical Sciences \& Sichuan Provincial People's \\ Hospital, 32 West Second Section, First Ring Road, Chengdu, 610072, Sichuan, China. Email: ph1726148853@qq.com.
}

Background: The incidence of placenta accreta spectrum (PAS) disorders has increased rapidly in recent years and is associated with several maternal and neonatal complications. Intravoxel incoherent motion (IVIM) imaging is a method which can assess placental perfusion quantitatively. Therefore, the first aim of this study was to investigate whether patients with adverse maternal and neonatal outcomes of PAS disorders differed in the parameters from IVIM. A second aim was to identify these parameters for adverse peripartum outcome in gravid patients at risk for PAS.

Methods: The subject group consisted of patients with placenta previa, in which 75 patients had PAS disorders and 24 patients did not have PAS disorders between 28+0 and 39+6 weeks, respectively. All women underwent magnetic resonance imaging (MRI) examination including an IVIM sequence with 8 b values on a 1.5 T scanner. The perfusion fraction (f), pseudodiffusion coefficient $\left(\mathrm{D}^{*}\right)$, and standard diffusion coefficient (D) were calculated. All medical records were received postpartum. The final degree of placental invasion was established either by placental villi alterations from a placental sample or from maternity records of the women's general practitioners.

Results: Women with PAS disorders had a higher perfusion fraction $(34.12 \%)$ than women without the disease $(29.39 \%)(\mathrm{P}<0.05)$. The perfusion fraction was $36.86 \%$ in women with massive blood loss and was $35.15 \%$ in women requiring transfusion, which was higher than women without massive blood loss and not requiring transfusion $(\mathrm{P}<0.05)$. The $\mathrm{D}$ value was $1.65 \times 10^{-3} \mathrm{~mm}^{2} / \mathrm{s}$ in women with low birth weight, which was lower than that in women with appropriate birth weight $\left(1.70 \times 10^{-3} \mathrm{~mm}^{2} / \mathrm{s}\right)(\mathrm{P}<0.05)$.

Conclusions: Patients with PAS disorders differed in placental perfusion fraction from women without PAS disorders. The $\mathrm{f}$ and $\mathrm{D}$ value may be used to recognize patients with certain adverse clinical outcomes.

Keywords: Placenta accreta spectrum disorders (PAS disorders); magnetic resonance imaging (MRI); placenta; intravoxel incoherent motion (IVIM); clinical outcome

Submitted Oct 31, 2019. Accepted for publication Dec 12, 2019.

doi: $10.21037 /$ tau.2019.12.27

View this article at: http://dx.doi.org/10.21037/tau.2019.12.27 


\section{Introduction}

The term placenta accreta spectrum (PAS) is the preferred umbrella term to define all grades of abnormal placentation, which include placenta accreta, increta, and percreta. The most common risk factors for PAS are placenta previa and previous cesarean delivery $(\mathrm{CD})$, with placenta previa being a significant independent risk factor for PAS. The UK national case-control study reported that the incidence of PAS was 577 per 10,000 births in women presenting with placenta previa and a prior CD (1). The combination of both placenta previa and PAS can result in high maternal morbidity and mortality at the time of delivery (2).

The most severe complication associated with PAS is hemorrhage, which can lead to multisystem organ failure, disseminated intravascular coagulation, hysterectomy, and, if the placenta does not detach completely from the uterus after delivery, death. The associated fetal and maternal mortality rate has been forecasted to reach $9-19 \%$ and $6-7 \%$ respectively $(3,4)$. More than half of women receive transfusions of blood products, and a third have incidental cystotomy in association with surgical management $(5,6)$. Studies also found an increased incidence of preterm delivery and of small-for-gestation age infants in pregnancies with PAS (7).

Multiple retrospective cohort studies of PAS disorders have revealed reduced maternal morbidity when cared for by multidisciplinary teams with expertise of this condition (8-11). Therefore, the antenatal diagnosis of PAS disorders is extremely important. Ultrasonography is a first line imaging modality for the detection of PAS. Magnetic resonance imaging (MRI) has been used increasingly in recent years and is preferred in the evaluation of a posterior placenta or in the detection of parametrial invasion. A recent systematic review reported that the sensitivity and specificity of MRI in diagnosing PAS varies between 75$100 \%$ and $65-100 \%$ respectively, but most of the studies analyzed were small sample size (12) and the diagnostic performance was usually high only when conducted by expert hands. Recent population studies have shown that, irrespective of the imaging modalities used, PAS disorders remain undiagnosed before delivery in half to two thirds of the cases $(1,13,14)$.

Intravoxel incoherent motion (IVIM) analysis is suitable for highly vascularized organs, it can assess placental perfusion quantitatively even without the use of contrast agents, and it also appears to be useful for evaluating placental microcirculation. From our previous study, we found placenta accreta and increta patients differ in placental perfusion fraction from women without the disease (15). However, we did not explore the association between IVIM parameters and adverse clinical outcomes of PAS disorders. Secondly, from Bourgioti's research, at least three MRI signs of PAS were strongly associated with complicated delivery and the presence of six or more MRI signs with major intraoperative complications (16). Despite the findings, there is a lack of clarity in this area, as the MRI features indicating PAS are subjective and the recognition of these features depends on the experience of the readers. The aim of this study was therefore to further investigate any association between IVIM parameters of the placenta and adverse maternal and neonatal clinical outcomes, in order to find a more objective parameter in evaluating the placenta in patients with PAS disorders. We hope this research can further aid in the effectiveness of individualized treatment for the patients with this complication.

\section{Methods}

The ethical review aboard approved the study, and informed consent was obtained from each woman participating in the study. Between January 2016 and October 2018, 206 gravid patients in the third trimester of pregnancy were referred for prenatal MRI, who were dedicated to placental evaluation; all patients were at risk for underlying PAS disorders due to the presence of placenta previa $(n=187)$, with ( $n=137)$ or without $(n=50)$ suspicious imaging findings on routine second trimester sonographic examination. Women with chronic hypertensions, pre-existing renal disease, and diabetes mellitus $(\mathrm{n}=11)$ were excluded. Women with inadequate surgical records $(n=75)$ or severe artifacts $(n=2)$ were also excluded. Ninety-nine patients completed the MR examination and formed our study group. The specific tomography of placental invasion was established in the operating room according to clinical and anatomical criteria. For patients who had total or subtotal hysterectomy, histologic criteria for PAS were based on chorionic villi attachment to the myometrium. For patients who did not require hysterectomy, the final diagnostic criteria of placental invasion used in this study included the following: during $\mathrm{CD}$, the placenta adhered firmly to the endometrium without invasion and showed non-selfcontrolled bleeding when detached; the placenta was deeply implanted in the myometrium and required curettage to remove invasive tissue; and the placenta villi penetrated through the uterine serosa or the surrounding anatomical structures. 


\section{Maternal and neonatal peripartum course}

Adverse maternal outcomes mainly included massive blood loss, hysterectomy, bladder reconstitution, and prolonged surgical times or ICU admission, and adverse neonatal outcomes included prematurity, low birth weight, and abnormal APGAR score. Estimated blood loss $\geq 2,000$ $\mathrm{mL}$ during surgery was defined as massive blood loss, birth at gestational week $\leq 36$ was defined as prematurity, birth weight $<2,500 \mathrm{~g}$ was defined as low birth weight, and APGAR score $<7$ at 5 min after birth was considered abnormal. Data concerning the onset of delivery (scheduled or acute), uterine preservation or hysterectomy, transfusion requirement, and admission to the intensive care unit were also collected. All obstetricians participating in the study agreed on the definitions of the above clinical terms, which were based on published data $(16,17)$.

\section{Imaging techniques}

MR imaging was performed on a $1.5 \mathrm{~T}$ scanner (Magnetom Aera, Siemens, Erlangen, Germany) using the integrated whole-body transmit-receive coil with six channels. The MRI protocol included examination from the diaphragm to the symphysis pubis using (I) axial, coronal, and sagittal halffourier acquisition single-shot turbo spin echo (HASTE) under the following parameters: field of view (FOV) $420 \times$ $80 \mathrm{~mm}, 5 \mathrm{~mm}$ thick section, $20 \%$ gap, matrix $272 \times 320$, scan duration time $50 \mathrm{~s}$; (II) axial, coronal and sagittal true fast imaging in steady-state precession ( TRUFISP) with the following parameters: an FOV of $420 \times 80 \mathrm{~mm}, 5 \mathrm{~mm}$ thick section, $30 \%$ gap, matrix $234 \times 384$, and a scan duration of $48 \mathrm{~s}$; (III) 3D-volumetric interpolated breath-hold examination (3D-VIBE) with the following parameters: FOV $400 \mathrm{~mm}$, $5 \mathrm{~mm}$ thick section, $20 \%$ gap, matrix $180 \times 320$, scan duration $8 \mathrm{~s}$; (IV) an IVIM sequence with 8 different b-values (0, $50,100,150,200,250,500,800 \mathrm{~s} / \mathrm{mm}^{2}$ ) was obtained perpendicular to the placenta. Forty slices, each of $5.5 \mathrm{~mm}$ thickness were collected. The matrix was $192 \times 120$, the FOV was $390 \times 304 \mathrm{~mm}$, and the NEX was 1 . Acquisition time for this sequence was typically 8 minutes and 37 seconds.

\section{Region of interest (ROI)}

Evaluation of the IVIM sequence was performed with research software (MITK diffusion). Two independent blinded observers, with 3 and 10 years of experience in obstetric imaging, carried out the measurement of IVIM.
In patients without PAS disorders, ROIs were placed in the middle part of the placenta including as large an area of the placenta as possible, but excluding areas with infarcts, hemorrhage, or other artifactual signal loss. The same ROIs were drawn on the slice above and below the middle slice. In patients with PAS disorders, the ROIs were placed in the regions of placental adhesion according to the maternity record after surgery, and the same ROIs were drawn on the slices above and below the middle slice. To avoid the flow artifacts in large vessels, ROIs should be located at least $2 \mathrm{~cm}$ from the insertion of the umbilical cord. Values of D, D*, and $\mathrm{f}$ were then calculated by averaging the total of the 3 ROIs.

\section{Statistical analysis}

Inter-rater reliability of the $\mathrm{f}, \mathrm{D}$, and $\mathrm{D}^{*}$ values between the 2 radiologists was assessed by the intra-class correlation coefficient. The normal distribution test was conducted in the variables. Physical and sociodemographic factors for PAS disorders were investigated by using the chi-square, student's $t$-test, or the Mann-Whitney u test to compare patients with PAS disorders to those without. The IVIM parameters were compared using Mann-Whitney u test. The IVIM parameters that could predict adverse maternal and neonatal events during delivery were calculated with the use of receiver operating characteristic curve analysis, and sensitivity and specificity values were determined for each of the optimal cutoffs. The correlation between IVIM parameters and the adverse maternal and neonatal events were evaluated by calculating Spearman's correlation coefficient $(\rho)$. P values $<0.05$ were considered statistically significant. All analyses were performed using IBM SPSS statistics 20.

\section{Results}

A total of 99 pregnant women with satisfactory raw images remained in the analysis (Figure 1). Intra-class correlation coefficient between 2 radiologists of the $\mathrm{f}, \mathrm{D}$, and $\mathrm{D}^{*}$ values were 0.892 (95\% CI: 0.839-0.928), 0.775 (95\% CI: $0.665-$ $0.849)$, and 0.541 (95\% CI: $0.317-0.629)$ respectively. The mean maternal age was $31.92 \pm 4.31$ years (range, $22-$ 41 years), and the mean gestational age at examination was 34 [4] weeks (range, 28-39 weeks). All gravid patients had placenta previa (complete, $n=70$; marginal, $n=22$; partial, $\mathrm{n}=4$; low lying, $\mathrm{n}=3$ ).

All medical records were received postpartum, 75 patients were diagnosed as PAS disorders, and 24 patients were diagnosed as without PAS disorders. The methods of 


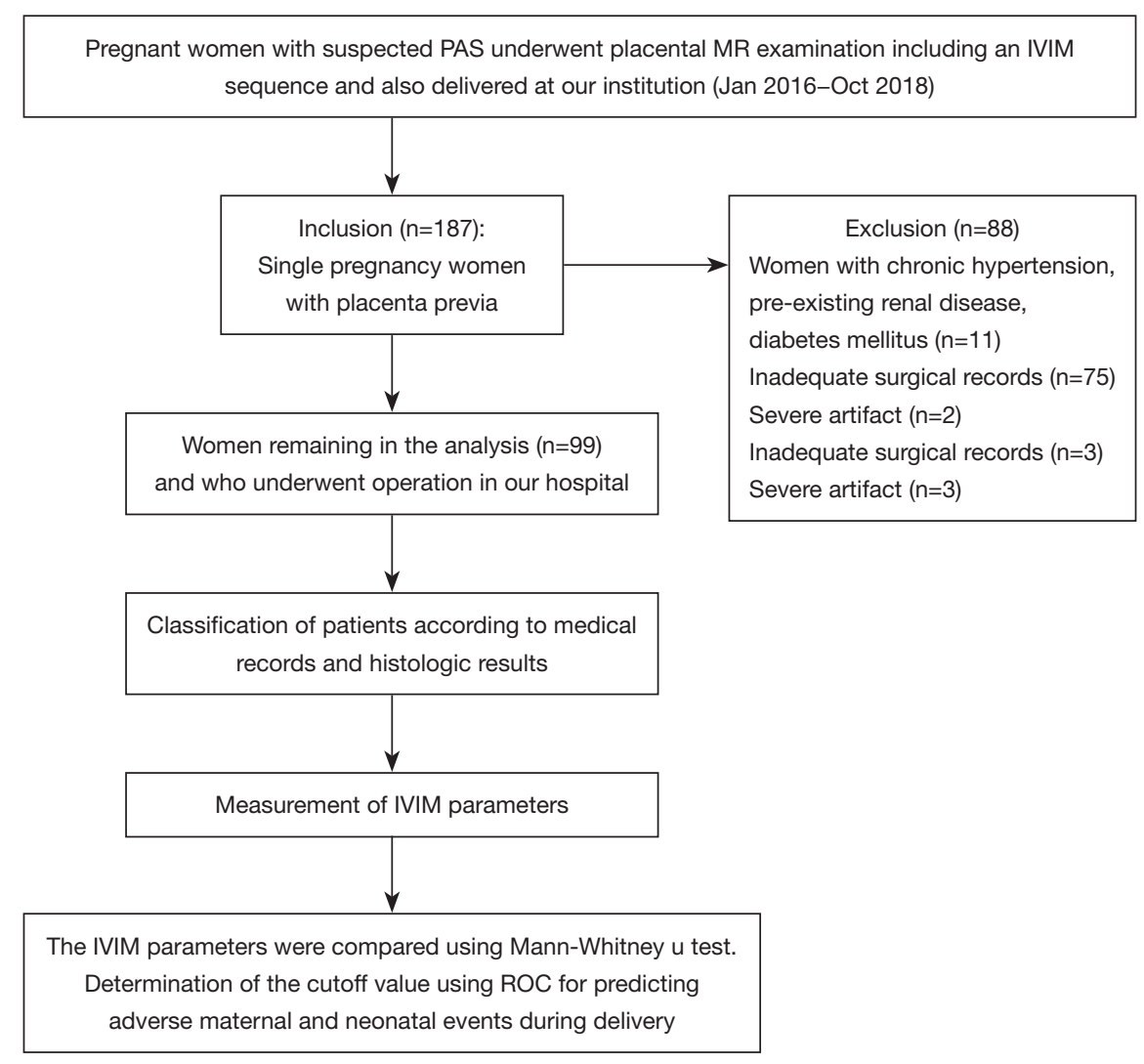

Figure 1 Flowchart of the study design. PAS, placenta accreta spectrum; IVIM, Intravoxel incoherent motion.

delivery included 50 cases of cesarean section, 45 cases of prophylactic distal abdominal aorta balloon occlusion and cesarean section, 2 cases of natural birth, 2 cases of total hysterectomy, and 1 case of partial hysterectomy. Table 1 presents the maternal characteristics of the study participants. The number of previous cesarean sections was greater in patients with PAS disorders than that in patients without the disease $(\mathrm{P}<0.05)$.

In 97 of 99 patients, delivery was scheduled. Mean estimated blood loss was 700 [500] mL. Blood loss $\geq 2,000 \mathrm{~mL}$ was recorded in $13 / 99$ patients. In total, 21 of 99 women were admitted to the ICU after delivery for careful monitoring (median $=3$ days, $\mathrm{QR}=2$ days). In total 38 of 99 patients received transfusion (median $=0, \mathrm{QR}=400 \mathrm{~mL}$ ). No maternal death was recorded. Preterm delivery was recorded in 34 of 99 cases, 22 of 99 newborns had low birth weight (mean $=2,758.84 \mathrm{~g}, \mathrm{SD}=434.13 \mathrm{~g}$ ), and 1 patient had a poor APGAR score (<7) 5 min after birth (Table 1).

When compared with patients with PAS disorders, f $(29.39 \%)$ and $D^{*}\left(20.37 \times 10^{-3} \mathrm{~mm}^{2} / \mathrm{s}\right)$ values were significantly lower in patients without the disease $(\mathrm{P}<0.05)$, while $\mathrm{D}$ values showed no statistical significance between the 2 groups $(\mathrm{P}>0.05)$. A comparison of IVIM parameters and adverse maternal and neonatal clinical outcomes between patients with PAS and patients without it is shown in Table 2. The blood loss during delivery in patients with PAS disorders was greater $(\mathrm{P}<0.05)$, and more patients with PAS disorders required transfusion and were admitted to the ICU $(\mathrm{P}<0.05)$. Gestational week at delivery, neonatal birth weight and $5 \mathrm{~min}$, and APGAR score showed no statistical difference between the 2 groups $(\mathrm{P}>0.05)$.

\section{Association of IVIM parameters with adverse maternal outcome}

Patients with massive blood loss and patients who required transfusion had a higher $\mathrm{f}$ value $(36.86 \%$ and $35.15 \%$ respectively) than patients without the adverse maternal outcome. However, D and $\mathrm{D}^{*}$ values did not show statistical difference between the 2 groups $(\mathrm{P}>0.05)$ (Table 2).

The optimal cutoff for predicting massive blood loss was the f value (32.37\%) with AUC $=0.647$ (95\% CI: 0.56-0.82, 
Table 1 Physical and sociodemographic features and adverse maternal and neonatal clinical outcomes of patients studied

\begin{tabular}{|c|c|c|c|}
\hline Variable & Patients without PAS disorders & Patients with PAS disorders & $P$ value \\
\hline Age (years) & $31.88 \pm 3.81$ & $31.93 \pm 4.48$ & 0.147 \\
\hline Less than 35 & $20(20.20 \%)$ & $51(51.52 \%)$ & \\
\hline 35 or older & $4(4.04 \%)$ & $24(24.24 \%)$ & \\
\hline \multicolumn{4}{|l|}{ Number of previous cesarean sections } \\
\hline 0 & $11(11.11 \%)$ & $15(15.15 \%)$ & \\
\hline 1 & $12(12.12 \%)$ & $46(46.46 \%)$ & \\
\hline 2 or more & $1(1.01 \%)$ & $14(14.14 \%)$ & 0.021 \\
\hline \multicolumn{4}{|l|}{ Previous uterine dilation and curettage } \\
\hline Blood loss (mL) & 500 [200] & $800[600]$ & 0.001 \\
\hline Transfusion & & & 0.003 \\
\hline No & $21(21.21 \%)$ & $40(40.40 \%)$ & \\
\hline Yes & $3(3.03 \%)$ & $35(35.35 \%)$ & \\
\hline $\begin{array}{l}\text { Gestational age at the time of delivery } \\
\text { (weeks) }\end{array}$ & $37[2]$ & $37[1]$ & 0.241 \\
\hline Birth weight of the neonates $(\mathrm{g})$ & $2,767.50 \pm 458.91$ & $2,756.67 \pm 429.08$ & 0.991 \\
\hline APGAR score, 5 min & $10[0]$ & $10[0]$ & 0.917 \\
\hline
\end{tabular}

PAS, placenta accreta spectrum.

$\mathrm{P}=0.037$ ), a sensitivity of $79 \%$, and a specificity of $54 \%$ (Figure 2). The optimal cutoff for predicting transfusion requirement was the $\mathrm{f}$ value $(31.97 \%)$ with $\mathrm{AUC}=0.657$ (95\% CI: $0.50-0.72, \mathrm{P}=0.009$ ), a sensitivity of $71 \%$, and a specificity $56 \%$ (Figure 3).

None of the IVIM parameters showed statistical difference in patients with the need for ICU stays.

\section{Association of IVIM parameters with adverse neonatal outcomes}

Patients with low birth weight had lower D value $(1.65 \times$ $10^{-3} \mathrm{~mm}^{2} / \mathrm{s}$ ) than patients without it, while $\mathrm{f}$ and $\mathrm{D}^{*}$ values did not show statistical difference between the 2 groups. None of the IVIM parameters showed statistical difference in patients with and without preterm delivery.

The optimal cutoff for predicting low birth weight was the $\mathrm{D}$ value $\left(1.70 \times 10^{-3} \mathrm{~mm}^{2} / \mathrm{s}\right)$ with $\mathrm{AUC}=0.672(95 \% \mathrm{CI}$ : $0.58-0.80, \mathrm{P}=0.013)$, a sensitivity of $74 \%$, and a specificity of $53 \%$ (Figure 4).

A positive correlation between $\mathrm{D}$ value and birth weight was also found $(\rho=0.23, P=0.025)$.

\section{Discussion}

The incidence of PAS has increased 10 fold in the past 50 years. The rapid increase in PAS can be directly linked to the steady increase in global CD rates. Additionally, more than $90 \%$ of women diagnosed with PAS also have a placenta previa. Consequently, our study mainly included 
Table 2 Association of IVIM parameters with adverse clinical outcomes ( $\mathrm{n}=99)$

\begin{tabular}{|c|c|c|c|c|}
\hline Variable & $\mathrm{N}(\%)$ & $f(\%)$ & $\mathrm{D}\left(10^{-3} \mathrm{~mm}^{2} / \mathrm{s}\right)$ & $\mathrm{D}^{*}\left(10^{-3} \mathrm{~mm}^{2} / \mathrm{s}\right)$ \\
\hline \multicolumn{5}{|c|}{ PAS disorders } \\
\hline No & $24(24.24)$ & 29.39 (5.59) & $1.68(0.13)$ & 20.37 (10.22) \\
\hline Yes & 75 (75.76) & $34.12(9.8)$ & $1.7(0.13)$ & $24.84(8.94)$ \\
\hline$P$ value & & 0.019 & 0.915 & 0.044 \\
\hline No & $86(86.87)$ & $31.98(10.38)$ & $1.70(0.13)$ & 22.61 (9.69) \\
\hline Yes & $13(13.13)$ & $36.86(8.64)$ & $1.67(0.15)$ & $28.37(9.58)$ \\
\hline$P$ value & & 0.025 & 0.234 & 0.063 \\
\hline \multicolumn{5}{|c|}{ Transfusion } \\
\hline$P$ value & & 0.009 & 0.715 & 0.021 \\
\hline \multicolumn{5}{|c|}{ Need of ICU stay } \\
\hline No & 78 (78.79) & 32.08 (9.32) & $1.69(0.12)$ & $25.21(12.67)$ \\
\hline Yes & $21(21.21)$ & 34.41 (11.95) & $1.69(0.17)$ & 26.33 (11.18) \\
\hline$P$ value & & 0.387 & 0.604 & 0.962 \\
\hline \multicolumn{5}{|c|}{ Preterm delivery } \\
\hline No & 65 (65.66) & $34.40(9.6)$ & $1.70(0.13)$ & $23.35(7.4)$ \\
\hline Yes & 34 (34.34) & $31.17(9.43)$ & $1.69(0.13)$ & $24.84(13.41)$ \\
\hline
\end{tabular}

IVIM, intravoxel incoherent motion; PAS, placenta accreta spectrum.

patients with placenta previa. When placenta previa and CD are present, the risk of PAS is even greater. In our study, 60 $(60.60 \%)$ patients with PAS disorders had previous cesarean deliveries, which was in accordance with the previous reports. PAS is often associated with massive blood loss requiring blood transfusion, and other clinical complications such as injury to surrounding organs, admission to ICU, and potential fetal compromise. In our study, patients with PAS also had greater blood loss and required more blood transfusion during delivery, and the number of patients admitted to ICU was also greater in patients with PAS.

Chantraine et al. (18) noted vessels were more heterogeneous in size and less uniformly distributed throughout the placenta bed in placenta increta; larger and smaller vessels were also identified from the placentalmyometrium junction. Millischer's contrast MRI findings also confirmed the hypervascular nature of the placenta bed in abnormally invasive placenta (19). These changes resulted in violent hemorrhage when the adherent placenta was removed, as these arteries conduct a far large volume of blood than the spiral arteries of smaller diameter. In our study, f value was significantly higher in patients with PAS disorders. This accounts for the particular vascular behavior of the invasive placenta, and explains the greater blood loss and more amount of transfusion that occurred in patients with PAS disorders. 


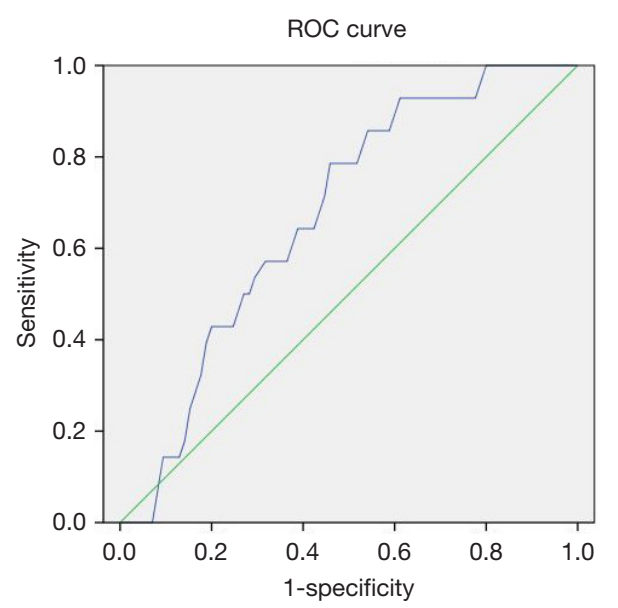

Figure 2 ROC curve of the f value for predicting massive blood loss.

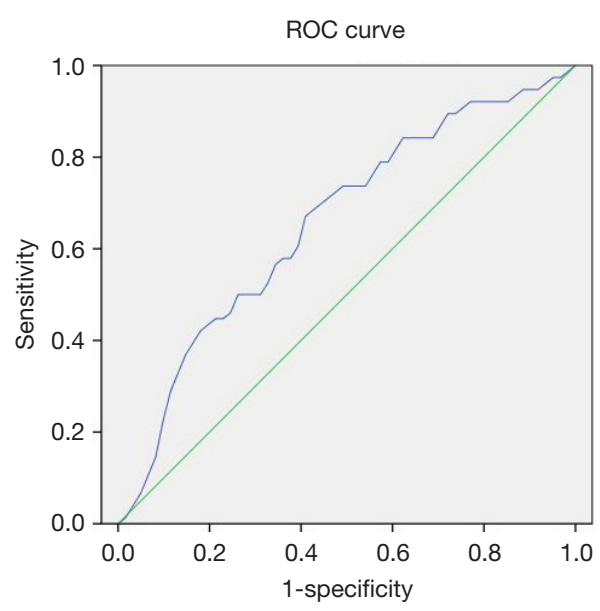

Figure 3 ROC curve of the $\mathrm{f}$ value for predicting requirement of transfusion.

When associating IVIM parameters with adverse maternal outcomes, we found the $\mathrm{f}$ value was significantly higher in patients with massive blood loss and in patients requiring transfusion of blood product. For placental imaging, $f$ is likely to represent the moving blood volume fraction compared with the total voxel volume $(20,21)$, and a higher $\mathrm{f}$ value represents a higher moving blood volume fraction. From our study, patients who had higher $\mathrm{f}$ value were more likely to suffer from severe hemorrhage during surgery and were also more likely to need transfusion due to the more hypervascular nature of the placental bed in these patients.

For a multidisciplinary team approach, not only should we recognize patients with PAS disorder prenatally, we

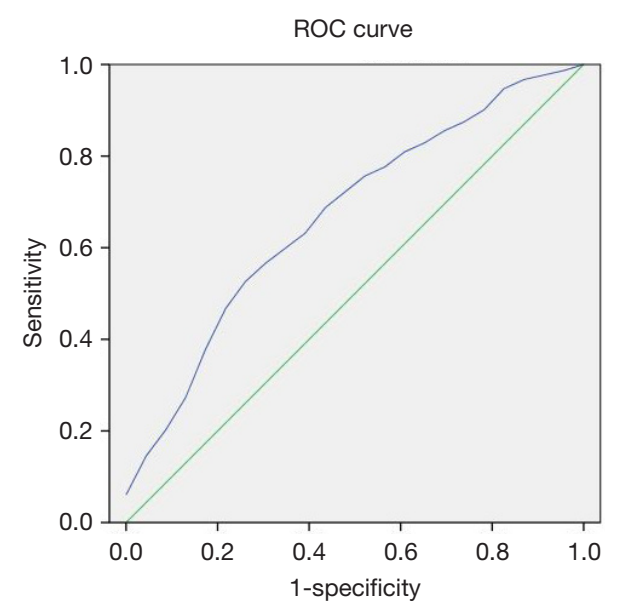

Figure 4 ROC curve of the D value for predicting low birth weight.

should also indicate whether there is a strong indication of massive blood loss, so that preparation of blood products and clotting factors and individualized delivery planning can be provided to achieve good postoperative outcomes and less maternal morbidity. For this consideration, we further demonstrated the cutoff of the $f$ value in predicting massive blood loss was $32.37 \%$, while it was $31.97 \%$ for deciding whether transfusion was required during delivery. The management can be more tailored if we are informed of potential massive hemorrhage in a timely manner and can then order blood for possible transfusion for a specific patient. The difference of IVIM parameters in patients with admission to the ICU was not detected in our study. This perhaps infers that admission to ICU is usually associated with acute onset of delivery, peripartum complications, and the surgical strategies applied, but is less associated with placental perfusion.

When associating IVIM parameters with adverse neonatal outcomes, we found the $\mathrm{D}$ value was significantly lower in patients with low birth weight than that in patients with normal birth weight neonates. The diffusion coefficient usually reflects cellular and interstitial characteristics of the tissue. The decrease in D in the placenta reflects the decrease of passive Brownian motion of water molecules in the interstitial spaces, resulting in the decreased transplacental exchanges. The placenta intermediates the transfer of nutrients between the mother and fetus, and thus the decreased levels of nutrients transporters may potentially influence the growth of the fetus. We also found a low correlation between the $\mathrm{D}$ value and the birth weight 
of the neonates. As a result, in patients with PAS disorders, it is possible that a lower $\mathrm{D}$ value represents a placental insufficiency in maternal-fetal exchanges. Therefore, we further identified that the best cutoff of $\mathrm{D}$ value to predict low birth weight was $1.70 \times 10^{-3} \mathrm{~mm}^{2} / \mathrm{s}$. The identification of patients with higher potential for low birth weight neonates can facilitate obstetrical management to include the administration of corticosteroid to the mother to enhance fetal pulmonary lung maturity. This can also offer information to neonatologists for the purpose of added care to neonates and hopefully, a decrease in neonatal morbidity. Secondly, no significant association of any IVIM parameters with preterm delivery was found; this is perhaps because most our patients had planned preterm deliveries.

Current studies about PAS have mainly focused on morphological changes of the placenta and their correlations with adverse clinical events (16,22). In China, although MRI is widely employed in obstetric-related imaging diagnosis, the prenatal diagnosis of PAS disorders remains subjective and requires expertise with the accuracy depending on the reader's experience, which is itself limited by the lack of both radiologists specialized in obstetric imaging and training programs for these radiologists. Consequently, there is a need for the objective evaluation of the PAS disorders, and for the prediction of the adverse clinical outcomes. With the use of IVIM, we can offer more objective information about the placental microcirculation in the multidisciplinary team discussion. We may indicate patients with possibility of massive blood loss and need of transfusion with the help of $f$ value, and indicate patients with the possibility of having low birth weight with the use of $\mathrm{D}$ value.

The results from our study were promising, but we still have to acknowledge several limitations of this study. First, we found that $f$ value was significantly higher in patients with PAS disorders, in patients with massive blood loss, and also in patients requiring transfusion. We also found $\mathrm{D}$ value was significantly lower in patients with low birth weight. However, when we tried to use $\mathrm{f}$ and $\mathrm{D}$ to predict adverse maternal and neonatal outcome, we found the diagnostic sensitivity and specificity of $\mathrm{f}$ and $\mathrm{D}$ were not high from our study. We assumed the biexponential fitting of IVIM was very sensitive to artifacts including respiratory motion, bowel movement, field inhomogeneity, magnetic susceptibility artifacts etc. Therefore, the parameters of IVIM may be influenced in this regard. Faster techniques and techniques less prone to signal loss from differences in magnetic susceptibility at air-tissue interfaces may help reduce this problem (23). Second, this is a retrospective study, not a prospective one. We mainly measured the areas of placenta invasion according to the description of the maternity record instead of measurement of the entire placenta. From our previous experience, IVIM parameters did not show statistical difference between the site where placenta separated from the uterine wall normally in the patients with placenta accreta and the regions of placenta in patients without placenta accreta (15). Chen et al. (24) also argued that future studies can focus only on abnormal high risk areas adjacent to the cesarean scar instead of the entire placenta. This may produce more predictive results, as more homogeneous regions adjacent to abnormal areas may skew the data. Third, our study population mainly included patients who already had a high risk of PAS disorders after ultrasonography, so the results may be biased as MRI is not a method used for screening. Fourth, the number of patients in this study is small, so the power of statistical analysis is possibly limited. However, our study can serve as a preliminary finding for further studies with larger sample sizes.

In conclusion, our study showed statistically significant differences between patients with PAS disorders and patients without the disease for the $f$ value, which may be due to the hypervascular nature of the placenta in patients with PAS. Our study also demonstrated that $f$ and $D$ values were associated with certain adverse maternal and neonatal outcomes, which could be used for better obstetrical management of these patients.

\section{Acknowledgments}

Funding: None.

\section{Footnote}

Conflicts of Interest: The authors have no conflicts of interest to declare.

Ethical Statement: The authors are accountable for all aspects of the work in ensuring that questions related to the accuracy or integrity of any part of the work are appropriately investigated and resolved. The ethical review aboard approved the study, and informed consent was obtained from each woman participating in the study.

Open Access Statement: This is an Open Access article distributed in accordance with the Creative Commons Attribution-NonCommercial-NoDerivs 4.0 International License (CC BY-NC-ND 4.0), which permits the noncommercial replication and distribution of the article with 
the strict proviso that no changes or edits are made and the original work is properly cited (including links to both the formal publication through the relevant DOI and the license). See: https://creativecommons.org/licenses/by-nc-nd/4.0/.

\section{References}

1. Fitzpatrick KE, Sellers S, Spark P, et al. Incidence and risk factors for placenta accreta/increta/percreta in the UK: a national case-control study. PLoS One 2012;7:e52893.

2. Green L, Knight M, Seeney FM, et al. The epidemiology and outcomes of women with postpartum haemorrhage requiring massive transfusion with eight or more units or red cells: a national cross-sectional study. BJOG 2016;123:2164-70.

3. Hull AD, Moore TR. Multiple repeat cesareans and the threat of placenta accreta: incidence, diagnosis, management. Clin Perinatol 2011;38:285-96.

4. Shweel M, Ameen NF, Zbrahiem MA, et al. Placenta accreta in women with prior uterine surgery: diagnostic accuracy of Doppler ultrasonography and MRI. Egypt J Radiol Nuclear Med 2012;43:473-80.

5. Eller AG, Porter TF, Soisson P, et al. Optimal management strategies for placenta accreta. BJOG 2009;116:648-54.

6. Warshak CR, Ramos GA, Eskander R, et al. Effect of predelivery diagnosis in 9 consecutive cases of placenta accreta. Obstet Gynecol 2010;115:65-9.

7. Gielchinsky Y, Mankuta D, Rojansky N, et al. Perinatal outcome of pregnancies complicated by placenta accreta. Obstet Gynecol 2004;104:527-30.

8. Shamshirsat AA, Fox KA, Salmamian B, et al. Maternal morbidity in patients with morbidity adherent placenta treated with and without a standardized multidisciplinary approach. Am J Obstet Gynecol 2015;212:218e1-218e9.

9. Al-Khan A, Gupta V, Ltlsley NP, et al. Maternal and fetal outcomes in placenta accreta after institution of teammanaged care. Reprod Sci 2014;21:761-71.

10. Eller AG, Bennett MA, Sharshiner M, et al. Maternal morbidity in cases of placenta accreta managed by a multidisciplinary care team compared with standard obstetric care. Obstet Gynecol 2011;117:331-7.

11. Smulian JC, Pascual AL, Hesham H, et al. Invasive placental disease: the impact of a multi-disciplinary team approach to management. J Matern Fetal Neonatal Med 2017;30:1423-27.

12. D'Antonio F, Lacovella C, Palacious-Jaraquemada J, et al. Prenatal identification of invasive placentation using magnetic resonance imaging: systemic review and metaanalysis. Ultrasound Obstet Gynecol 2014;44:8-16.
13. Bailit JL, Grobman WA, Rice MM, et al. Morbidity adherent placenta treatment and outcomes. Obstet Gynecol 2015;125:683-9.

14. Thurn L, Lindqvist PG, Jarobsson M, et al. Abnormally invasive placenta prevalence, risk factors and antenatal suspicion: results from a large population-based pregnancy cohort study in the Nordic countries. BJOG 2016;123:1348-55.

15. Lu T, Pu H, Cui $W$, et al. Use of intravoxel incoherent motion MR imaging to assess placental perfusion in patients with placental adhesion disorder on their third trimester. Clinical imaging 2019;56:135-9.

16. Bourgroti C, Zafeiropoulou K, Fotopoulos S, et al. MRI prognostications for adverse maternal and neonatal clinical outcome in patients at high risk for placenta accreta spectrum (PAS) disorders. Journal of MRI 2018;50:602-18.

17. Lim G, Horowitz JM, Berggruen S, et al. Correlation of probability scores of placenta accreta on magnetic imaging with hemorrhagic morbidity. J Clin Anesth 2016;34:261-9.

18. Chantraine F, Blacher S, Berndt S, et al. Abnormal vascular architecture at the placental-maternal interface in placenta increta. Am J Obstet Gynecol 2012;207:188.e1-9.

19. Millischer AE, Deloison B, Silvera S, et al. Dynamic contrast enhanced MRI of the placenta: a tool for prenatal diagnosis of placenta accreta. Placenta 2017;53:40-7.

20. Gowland P. Placenta MRI. Semin Fetal Neonatal Med 2005;10:485-90.

21. Moore RJ, Issa B, Tokarczuk R, et al. In vivo intravoxel incoherent motion measurements in the human placenta using echo-planar imaging at $0.5 \mathrm{~T}$. Magn Reson Med 2000;43:295-302.

22. Chen T, Xu XQ, Shi HB, et al. Conventional MRI features for predicting the clinical outcome of patients with invasive placenta. Diagn Interv Radiol 2017;23:173-9.

23. Sohlberg S, Mulic-Lutvica A, Lindgren P, et al. Placenta perfusion in normal pregnancy and early and late preeclampsia: a magnetic resonance imaging study. Placenta 2014;35:202-6.

24. Chen E, Mar MA, Horowitz JM, et al. Texture analysis of placental MRI: can it aid in the prenatal diagnosis of placenta accreta spectrum? Abdom Radiol (NY) 2019;44:3175-84.

Cite this article as: $\mathrm{Lu}$ T, Song B, Pu H, Li KD, Huang MW, Mei J, Wang SY. Prognosticators of intravoxel incoherent motion (IVIM) MRI for adverse maternal and neonatal clinical outcomes in patients with placenta accreta spectrum disorders. Transl Androl Urol 2020;9(2):258-266. doi: 10.21037/ tau.2019.12.27 